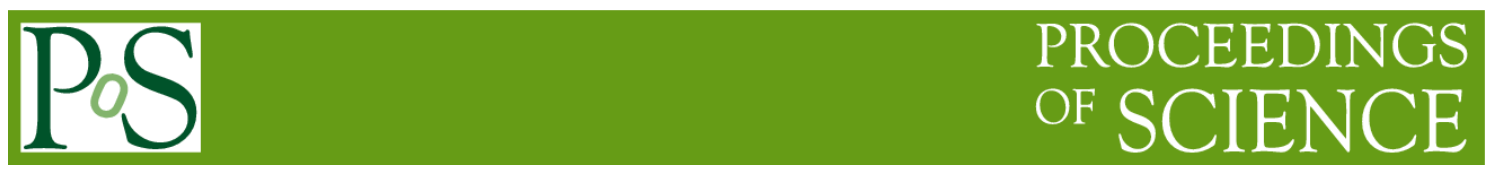

\title{
Top Physics in ATLAS and CMS
}

\author{
Jin Wang ${ }^{1}$ \\ Institute of High Energy Physics, Chinese Academy of Sciences \\ $19 B$ Yuquan Road, Shijingshan District, Beijing City, China \\ E-mail: wangjinecern.ch
}

Top physics results are one of the core physics deliveries in ATLAS and CMS experiments at the Large Hadron Collider (LHC) at CERN. This paper presents the most recent results on top quark production and top property measurements, as well as new physics searches with top events from ATLAS and CMS. All of which use the proton-proton collision data collected with LHC at centerof-mass energies of $8 \mathrm{TeV}$ and $13 \mathrm{TeV}$.

Corfu Summer Institute 2019 "School and Workshops on Elementary Particle Physics and Gravity" (CORFU2019)

31 August - 25 September 2019

Corfü, Greece

\section{${ }^{1}$ Speaker}




\section{Introduction}

Top quark is very unique as it is the most massive known fundamental particle, and the only quark that decays before hadronization. It gives opportunity to study a bare quark and access to its spin and polarization. Top quark plays a special role in mechanisms of the electroweak symmetry-breaking (EWSB), and it is expected to couple to new resonances predicted by a range of new physics models. The precise measurements of top quark properties and its productions are crucial to testing the Standard Model (SM) predictions and to searching physics beyond the Standard Model (BSM).

The Large Hadron Collider (LHC) at CERN is a top quark factory and it produces more than 100 million top quarks in its second run from 2015 to 2018 (Run 2). ATLAS and CMS are two general purpose detectors located at the LHC and the latest results on top physics from ATLAS and CMS experiments will be discussed here.

\section{Top quark production measurements}

\section{1 $t \bar{t}$ inclusive and differential cross sections}

$t \bar{t}$ production cross section measurements provide important tests on QCD model with massive partons and help to constrain SM parameters and PDFs, as well as anomalous Effective Filed Theory (EFT) terms. Figure 1 shows the summary of the $t \bar{t}$ inclusive cross section measurements from ATLAS and CMS with proton-proton collision data at centre-of-mass energies of 7,8 and $13 \mathrm{TeV}$. CMS has published a recent measurement [1] with $t \bar{t} \rightarrow$ $\left(l v_{l}\right)\left(\tau_{h} v_{\tau}\right) b \bar{b}$ final state, giving $\sigma_{t \bar{t}}=781 \pm 7$ (stat) \pm 62 (syst) \pm 20 (lumi) pb. The ratio of the partial width to the total width is also presented $\Gamma\left(t \rightarrow \tau v_{\tau} b\right) / \Gamma_{\text {total }}=0.1050 \pm$ 0.0009 (stat) \pm 0.0071 (syst). For the inclusive $t \bar{t}$ production cross section measurement, the latest results from ATLAS [2] used $36.1 \mathrm{fb}^{-1}$ proton-proton collision data at $13 \mathrm{TeV}$ accumulated in 2015 and 2016 and the cross section of $t \bar{t}$ measured is $\sigma_{t \bar{t}}=826.4 \pm$ 3.6 (stat) \pm 11.5 (syst) \pm 15.7 (lumi) \pm 1.9 (beam) $p b$, which reaches $2.4 \%$ precision. All these $t \bar{t}$ production cross section results show good agreement between data and the theoretical predictions.

$t \bar{t}$ differential cross section measurements scrutinize $t \bar{t}$ production in many channels as a function of different observables. They could give precision tests of QCD in different regions of phase space and they are sensitive to BSM physics. Figure 2 and Figure 3 shows the results of $t \bar{t}$ differential cross section measurements from ATLAS [2][3] and CMS [4], where most of the results are consistent with Next-to-Leading-Order (NLO) QCD predictions. The data in CMS results shows softer top $\mathrm{p}_{\mathrm{T}}$ than POWHEG+PYTHIA predicted in dilepton channel and other variables related to top $\mathrm{p}_{\mathrm{T}}$ are also in tension. Both ATLAS and CMS explored finer diferential regions of the $t \bar{t}$ cross section and an example of CMS results [5] can be found in Figure 4.

\subsection{Single top quark production cross sections}

The single top quark productions are electroweak top quark productions at the LHC and they provide a directory probe of the CKM matrix element Vtb. There are 3 channels, $t$-channel, $t W$ channel and s-channel. Figure 5 gives a summary of the single top quark production measurement 
of these 3 channels in ATLAS and CMS. The Run 1 combination of ATLAS and CMS single top quark measurements is used to extract the CKM matrix element Vtb [6] and the results are shown in Figure 6.

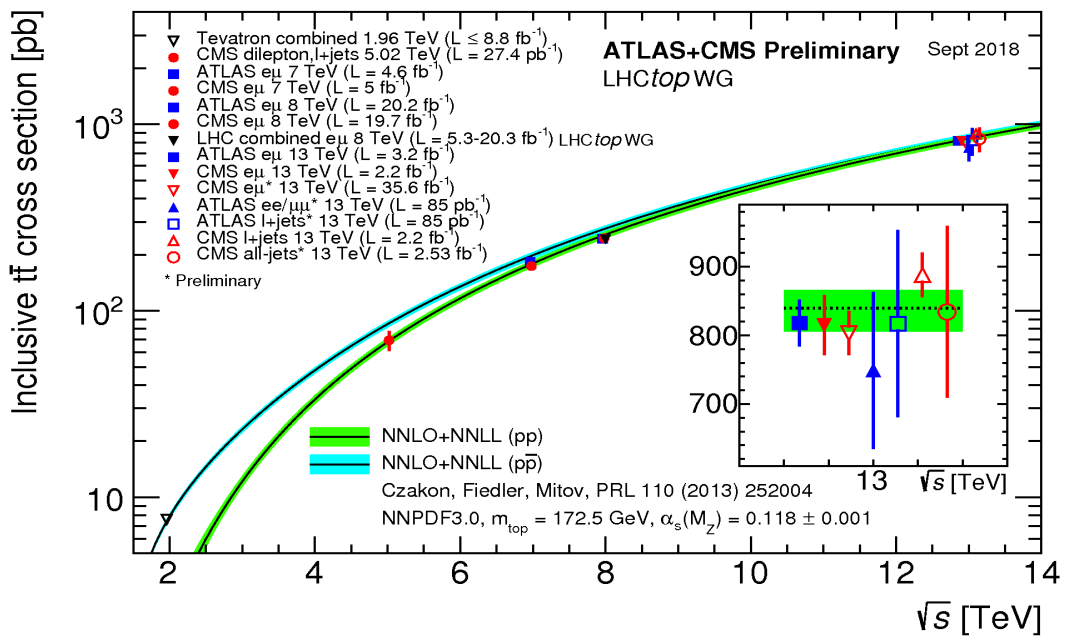

Figure 1: Summary of LHC and Tevatron measurements of the top-pair production crosssection as a function of the centre-of-mass energy compared to the NNLO QCD calculation complemented with NNLL resummation (top++2.0). The theory band represents uncertainties due to renormalisation and factorisation scale, parton density functions and the strong coupling. The measurements and the theory calculation are quoted at $m_{\text {top }}=172.5 \mathrm{GeV}$. Measurements made at the same centre-of-mass energy are slightly offset for clarity.

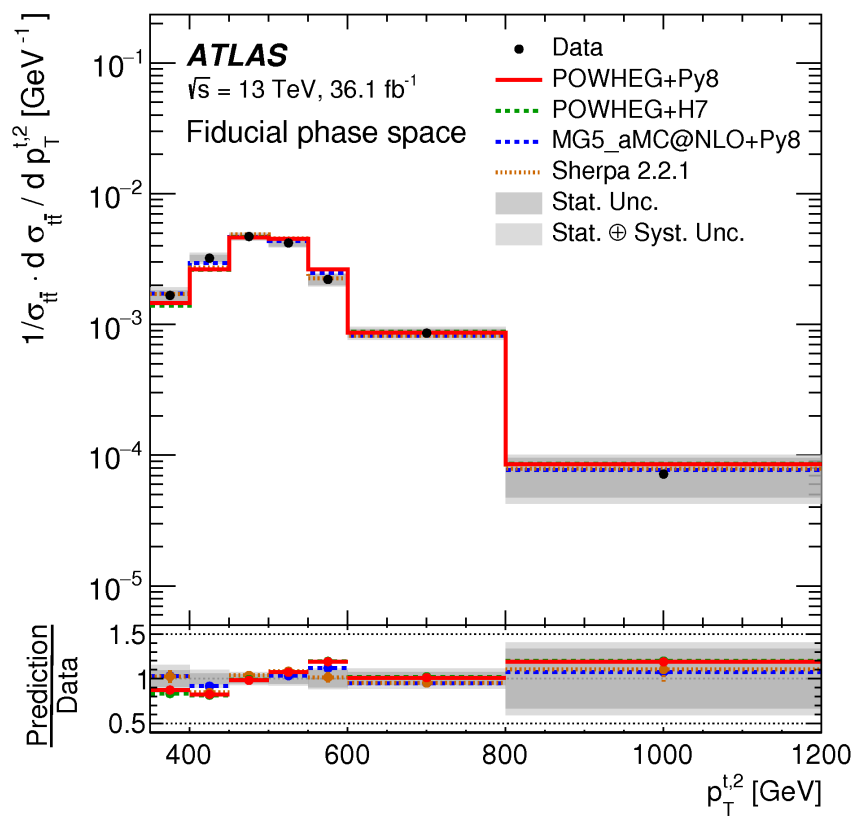

Figure 2: Normalized particle-level fiducial phase-space differential cross-sections as a function of transverse momentum of the second-leading top-quark jet. The gray bands indicate the total uncertainty in the data in each bin. The vertical bars indicate the statistical uncertainties in the theoretical models. The Powheg+Pythia8 generator is used as the nominal prediction. Data points are placed at the center of each bin. 


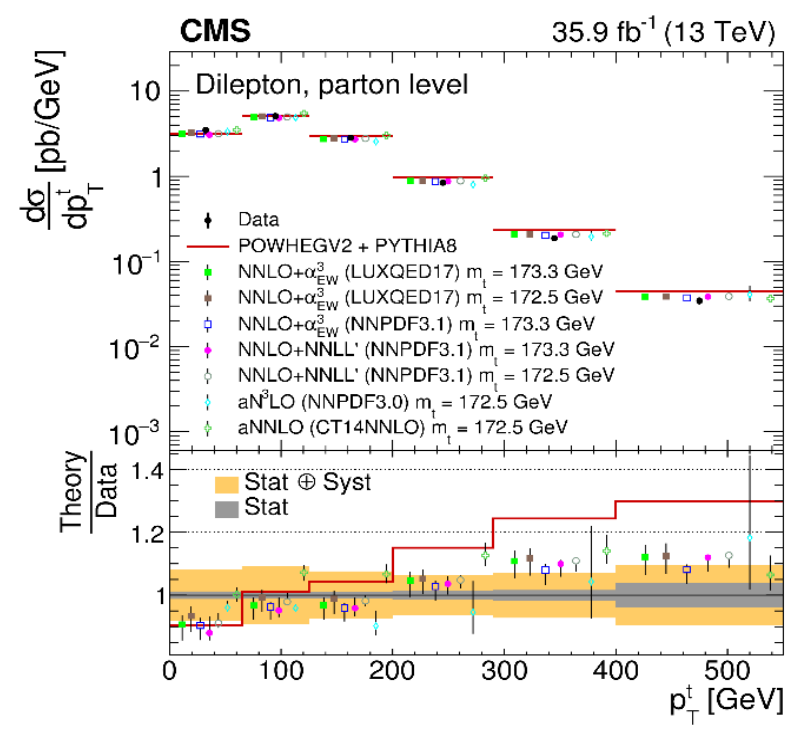

Figure 3: The differential $t \bar{t}$ production cross sections as a function of $p_{T}^{t}$ are shown for the data (filled circles), the theoretical predictions with beyond-NLO precision (other points) and the prediction from POWHEG+PYTHIA (solid line). The vertical lines on the filled circles and other points indicate the total uncertainty in the data and theoretical predictions, respectively. The left and right plots correspond to absolute and normalised measurements, respectively. The lower panel in each plot shows the ratios of the theoretical predictions to the data. The dark and light bands show the relative statistical and total uncertainties in the data, respectively.

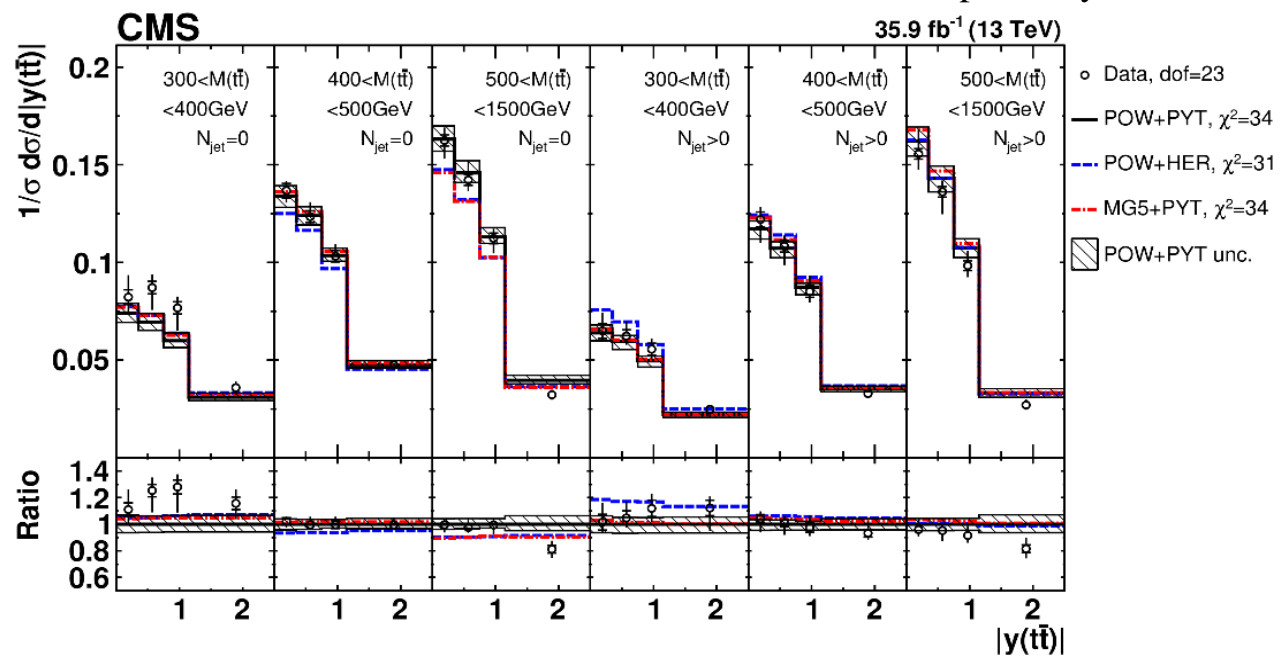

Figure 4: Comparison of the measured $\left[N_{\text {jet }}^{0,1+}, M(t \bar{t}), y(t \bar{t})\right]$ cross sections to the theoretical predictions calculated using POWHEG + PYTHIA ('POW+PYT'), POWHEG + HERWIG++ ('POW+HER'), and MG5 _aMC@NLO + PYTHIA ('MG5+PYT') event generators.\} The inner vertical bars on the data points represent the statistical uncertainties and the full bars include also the systematic uncertainties added in quadrature. For each MC model, values of $\chi^{2} \chi^{2}$ which take into account the bin-to-bin correlations and dof for the comparison with the data are reported. The hatched regions correspond to the theoretical uncertainties in POWHEG + PYTHIA. In the lower panel, the ratios of the data and other simulations to the 'POW+PYT' predictions are shown. 


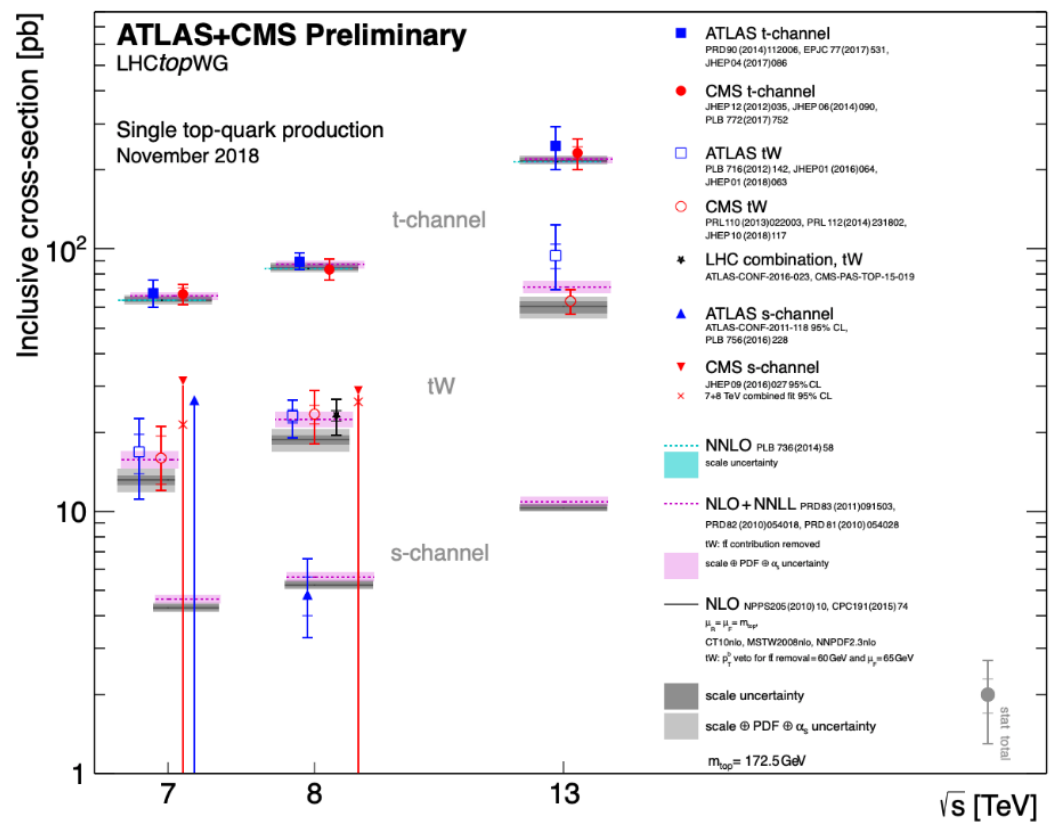

Figure 5: Summary of ATLAS and CMS measurements of the single top production crosssections in various channels as a function of the center of mass energy. The measurements are compared to theoretical calculations based on: NLO QCD, NLO QCD complemented with NNLL resummation and NNLO QCD (t-channel only).

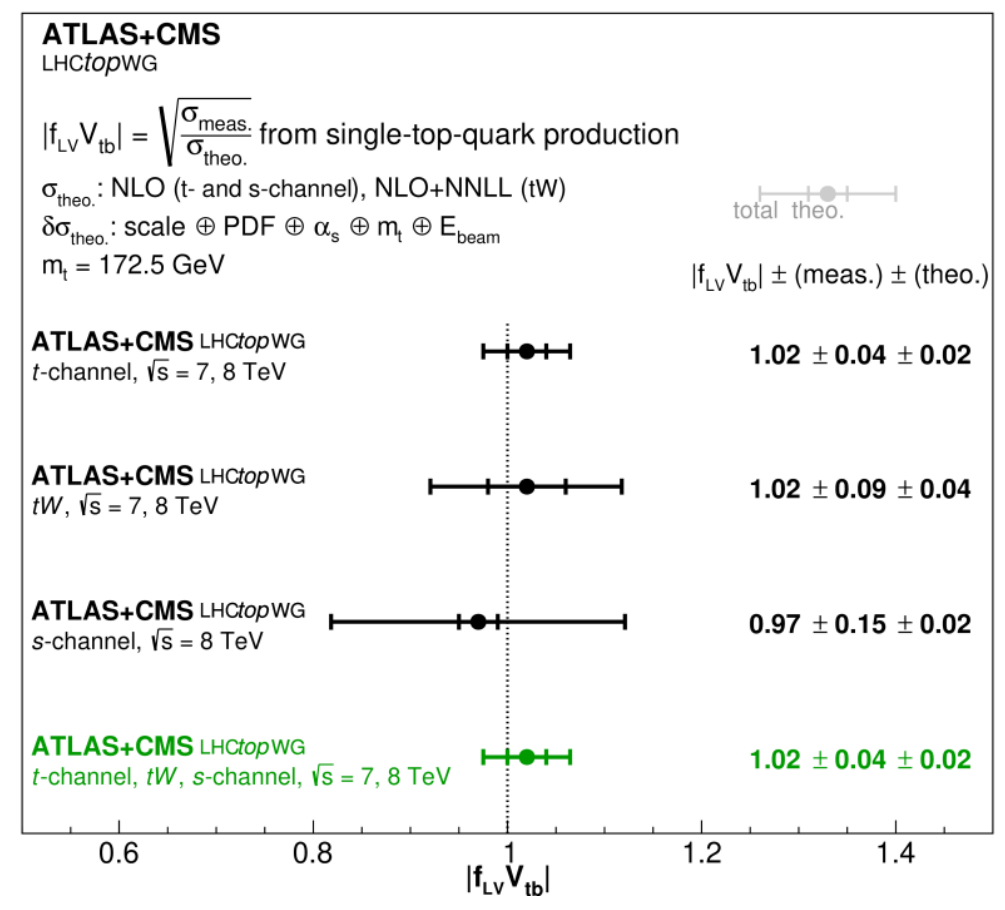

Figure 6: The combined $\left|f_{L V} V_{t b}\right|$ value extracted from the tt-channel and tW cross-section measurements at $\sqrt{s}=7$ and $8 \mathrm{TeV}$ from ATLAS and CMS, as well as the ATLAS ss-channel measurement at $\sqrt{s}=8 \mathrm{TeV}$, is shown together with the combined $\left|f_{L V} V_{t b}\right|$ values for each production mode. The theoretical predictions for tt-channel and ss-channel production are computed at NLO accuracy, while the theoretical predictions for tW are calculated at NLO+NNLL accuracy. The oteooteo uncertainties used to compute $\left|f_{L V} V_{t b}\right|$ include scale, PDF $+\alpha_{s}, m_{t}$, and $E_{\text {beam }}$ variations. 


\subsection{Top $+\mathrm{X}$ and rare top production measurements}

Many top $+X$ productions are becoming accessible with increasing data. In 2019, CMS published the first observation of the tZq production [7] with binned maximum likelihood fits to BDTs of three signal regions and the WZ/ZZ control regions as shown in Figure 7, using 77.4 $f b^{-1}$ proton proton collision data at $13 \mathrm{TeV}$. The cross section is measured with $15 \%$ precision $\sigma\left(t Z q \rightarrow t l^{+} l^{-} q\right)=111 \pm 13$ (stat) ${ }_{-9}^{+11}$ (syst) $\mathrm{fb}$. ATLAS observed first evidence of this process with observed (expected) significance of 4.2 (5.4) $\sigma$, using $36 \mathrm{fb}^{-1}$ data [8].

The measurements of $\sigma(t t Z)$ and $\sigma(t t \gamma)$, as well as their first differential cross sectionresults are reported by CMS in [9] and ATLAS in [10] respectively. Evidence of $t \gamma q$ production are found by CMS in 2018 with observed (expected) significance 4.4 (3.0) $\sigma$. Both ATLAS and CMS searched for the rare four top production, whose production cross section is tiny in SM but could be significantly increased in BSM scenarios. The results from ATLAS using $36 \mathrm{fb}^{-1}$ data [11] are shown in Figure 8. CMS used full Run 2 data and fitted data with BDT distribution of signal and background models, as shown in Figure 9. The CMS results yield an observed (expected) signal significance of 2.6 (2.7) $\sigma$ [12].

The $t \bar{t}+b b$ production is important background to $t t H, H \rightarrow b b$ study. In ATLAS and CMS, different phase spaces of $t \bar{t}+b b$ process are compared to NLO MC simulations, as shown in Figure 10 and Figure 11. small tensions between the data and prediction are observed in both ATLAS [13] and CMS [14].
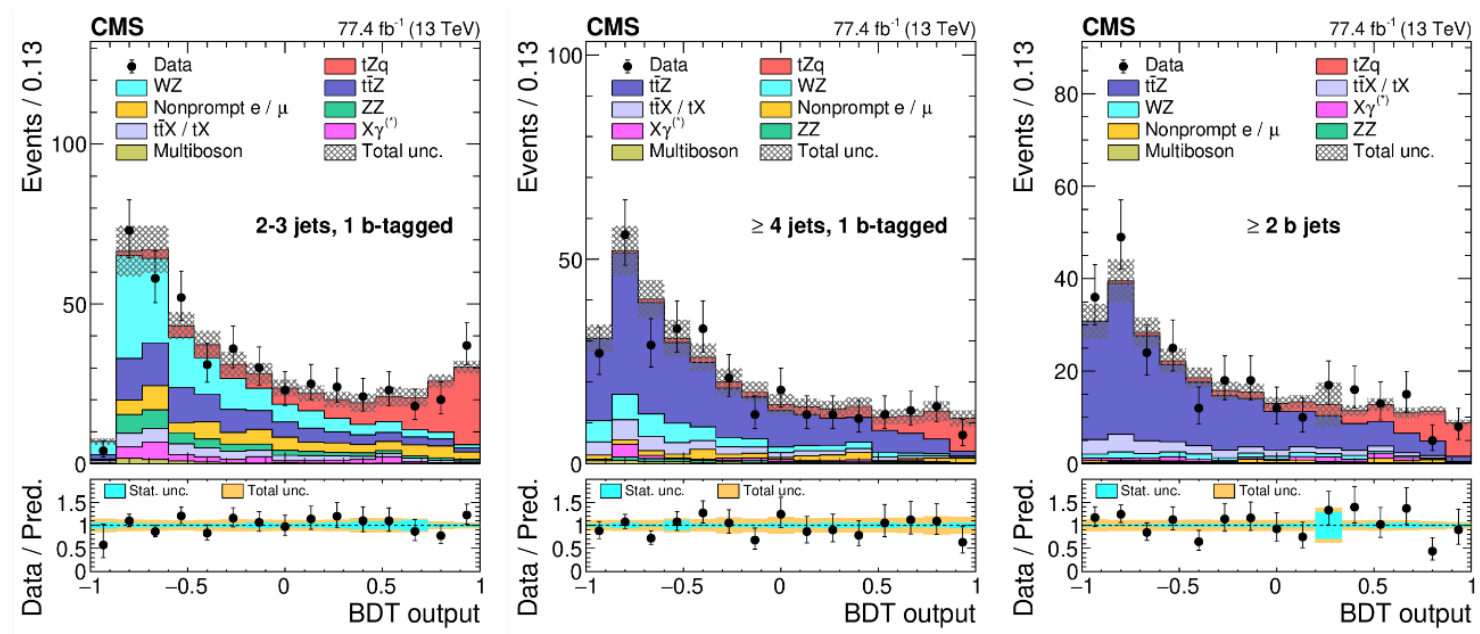

Figure 7: Observed (points) and post-fit expected (shaded histograms) BDT distributions for events in SR-2/3j-1b (left), SR-4j-1b (middle), and SR-2b (right). The vertical bars on the points represent the statistical uncertainties in data. The hatched regions show the total uncertainties in the background. The lower panels display the ratio of the observed data to the predictions, including the tZqtZq signal, with inner and outer shaded bands, respectively, representing the statistical and total uncertainties in the predictions. 


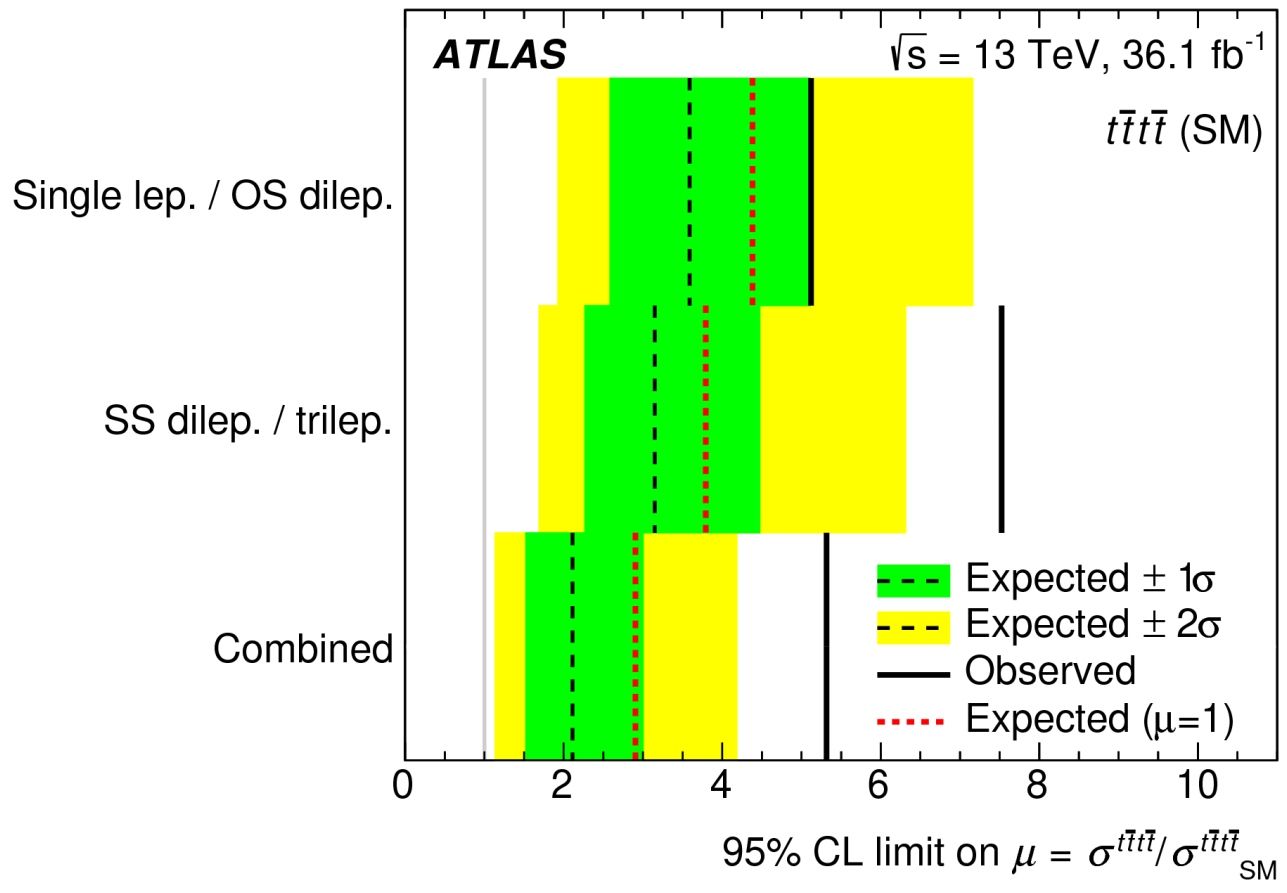

Figure 8: Summary of the 95\% CL upper limits on $\sigma(\mathrm{tt} \overline{\mathrm{t}})$ relative to the SM prediction in the individual channels and for the combination. The observed limits (solid black lines) are shown together with the expected limits in the background-only hypothesis (dashed black lines) and in the SM signal-plus-background hypothesis case (dashed red lines). One- and two-standarddeviation uncertainty bands around the expected limits in the background-only hypothesis are also shown.

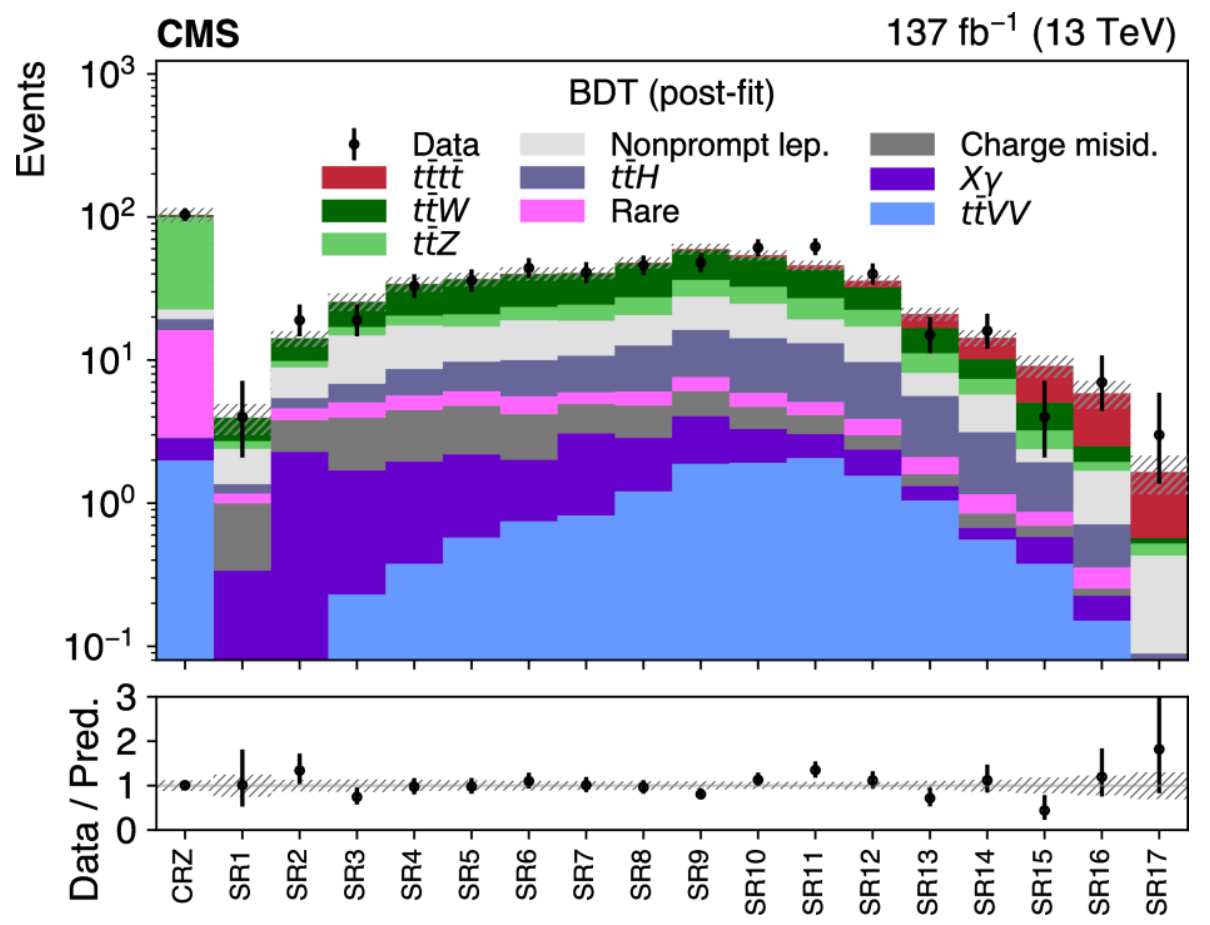

Figure 9: Observed yields in the control and signal regions for the BDT analysis, compared to the post-fit predictions for signal and background processes. The hatched areas represent the total post-fit uncertainties in the signal and background predictions. The lower panel shows the ratios of the observed event yield to the total prediction of signal plus background. 


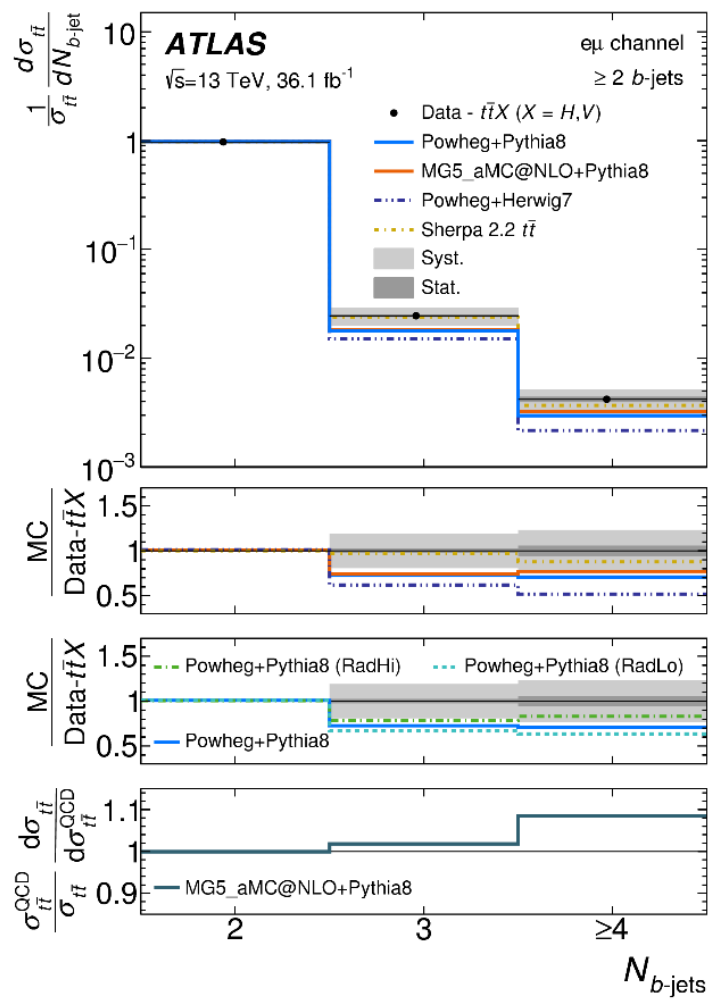

Figure 10: The relative differential cross-section as a function of the b-jet multiplicity in events with at least two b-jets in the e $\mu$ channel compared with various MC generators. The t $\bar{t} H$ and $\mathrm{t} \overline{\mathrm{t}} \mathrm{V}$ contributions are subtracted from data. Three ratio panels are shown, the first two of which show the ratios of various predictions to data. The third panel shows the ratio of predictions of normalised differential cross-sections from MGMCatNLO+PYTHIA 8 including (numerator) and not including (denominator) the contributions from $t \overline{\mathrm{t}} \mathrm{V}$ and $\mathrm{t} \overline{\mathrm{t}} \mathrm{H}$ production. Uncertainty bands represent the statistical and total systematic uncertainties as described in the text.
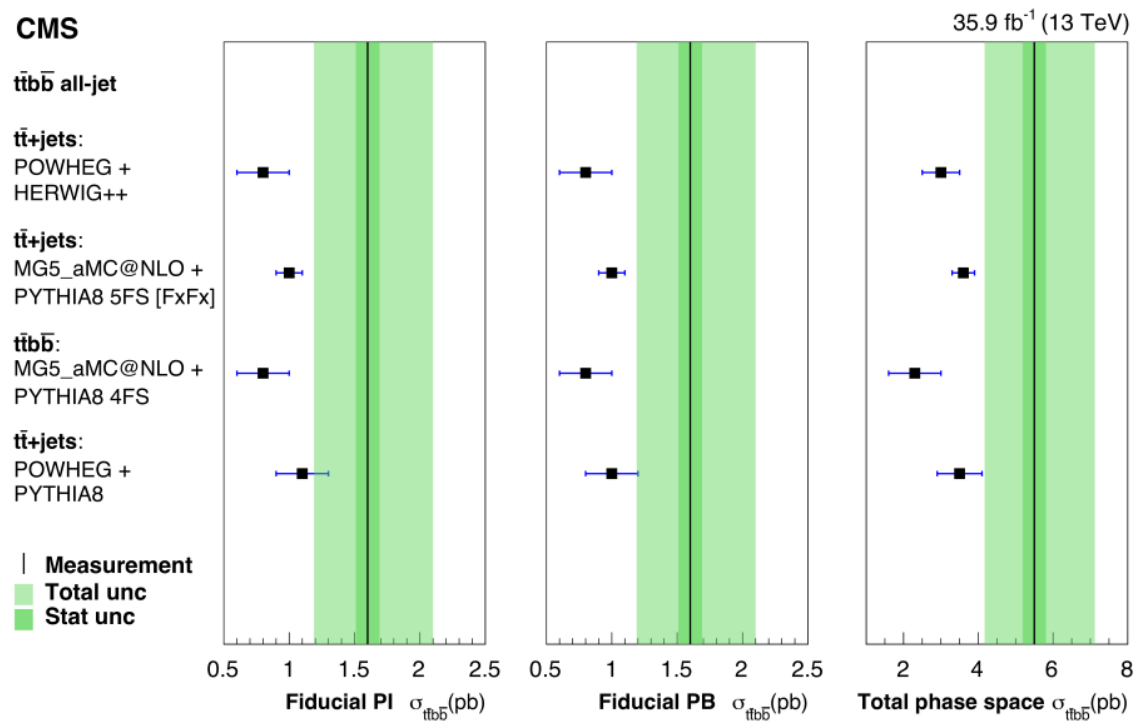

Figure 11: Comparison of the measured $t \bar{t} b \bar{b}$ production cross sections (vertical lines) with predictions from several Monte Carlo generators (squares), for three definitions of 
our $t \bar{t} b \bar{b}$ regions of phase space: fiducial PI (left), fiducial PB (middle), total (right). The dark (light) shaded bands show the statistical (total) uncertainties in the measured value. Uncertainty intervals in the theoretical cross sections include the statistical uncertainty as well as the uncertainties in the PDFs and the $\mu_{R}$ and $\mu_{F}$ scales.

\section{Top quark property measurement}

\subsection{Top mass measurement}

Top quark mass is a key parameter of the Standard Model and it is important for electroweak vacuum stability. The top mass needs to be measured in all possible ways with highest possible precision. One way is the direct measurement of "Monte Carlo mass" $m_{t}^{M C}$, extracted from invariant mass of decay products. Another way is indirect measurement of the pole mass $m_{t}^{\text {pole }}$, from observables depending on $m_{t}$, e.g. inclusive or differential cross section compared to different theoretical predictions. Difference between $m_{t}^{M C}$ and $m_{t}^{\text {pole }}$ could be $\sim \mathrm{GeV}$.

In 2018, ATLAS updated the direct top mass measurement using lepton+jets channel with $20.2 \mathrm{fb}^{-1}$ data collected at $8 \mathrm{TeV} m_{t}^{M C}=172.08 \pm 0.39$ (stat) \pm 0.82 (syst) $\mathrm{GeV}$ [15]. CMS also published the direct top mass measurement with lepton+jets channel using $36 \mathrm{fb}^{-1}$ data collected at $13 \mathrm{TeV}$ in $2016 m_{t}^{M C}=172.25 \pm 0.08$ (stat + JSF) \pm 0.62 (syst) GeV [16]. A summary of direct top mass measurements from ATLAS and CMS can be found in Figure 12, with the best precision reaches $\sim 0.5 \mathrm{GeV}$.

Indirect measurement of the pole mass $m_{t}^{\text {pole }}$ are also extracted from differential measurement in both ATLAS [17] and CMS [5]. The results from CMS that give most precise results to date are shown in Figure 13. CMS also presented a measurement of top mass from boosted jet mass observable, using reconstruct highly-boosted top quark decays with a novel $\mathrm{XCone}$ jet algorithm. In this analysis, the normalized differential cross section as a function of jet mass is compared to predictions from POWHEG with different values of top mass. The top mass is extracted to be $m_{t}^{\text {pole }}=172.56 \pm 2.47 \mathrm{GeV}$.

The evolution of the top quark mass as a function of the scale could be extracted by comparing differential $t \bar{t}$ production cross section as a function of $m_{t \bar{t}}$ at the parton level, to NLO predictions in the $\overline{M S}$ scheme obtained with different values of $m_{t}$. CMS obtained the first results of the running of the top quark mass measurement [19], as shown in Figure 14. The data shows good agreement to theoretical predictions.

\subsection{Top pair spin correlations}

Top quarks in $t \bar{t}$ production are mainly unpolarized, but the top pairs are strongly correlated. Some BSM scenarios would lead to different top spin correlation. Leptons from top decay carry the most spin information of the parent top and the easiest observable to probe top spin correlations would be the azimuthal opening angle $\Delta \varphi$ between $l^{+} l^{-}$. In ATLAS and CMS, unfolded parton-level differential cross sections for $\Delta \varphi\left(l^{+} l^{-}\right)$are compared to different generator predictions [20][21], as shown in Figure 15 and Figure 16, respectively. Stronger spin correlation is observed in data comparing to NLO predictions. 


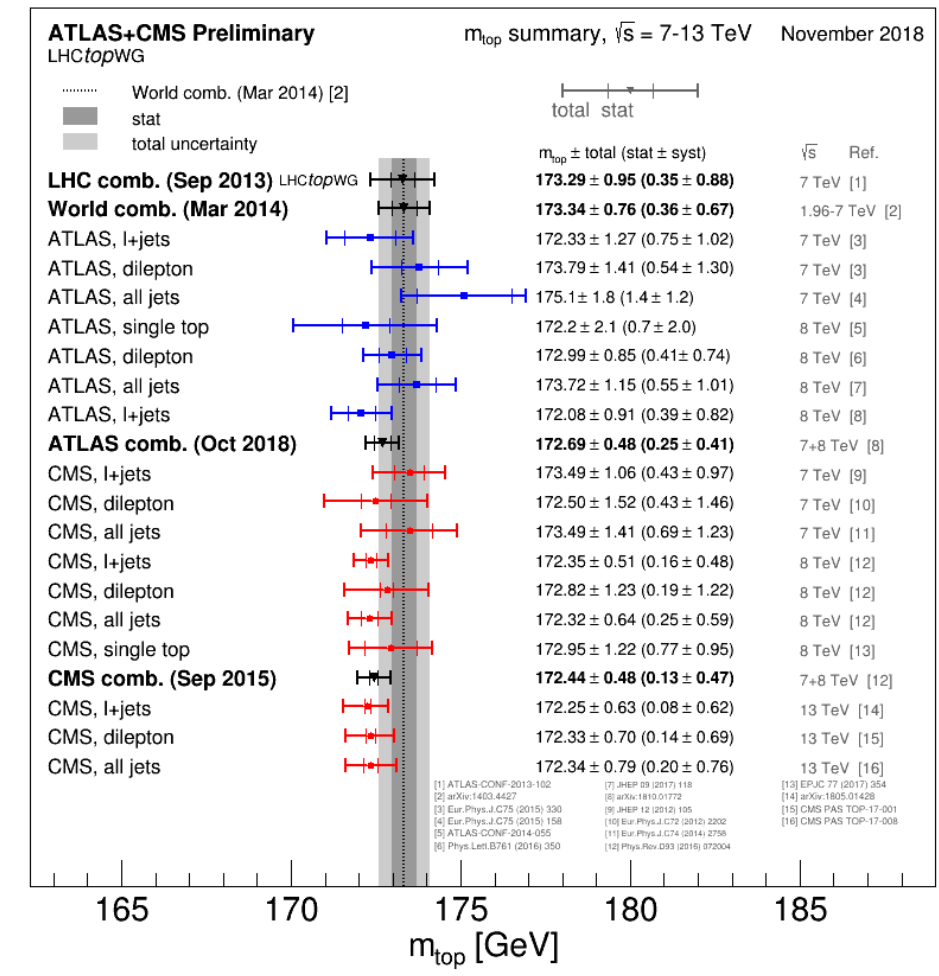

Figure 12: Summary of the ATLAS and CMS direct mtop measurements. The results are compared with the LHC and Tevatron+LHC mtop combinations. For each measurement, the statistical uncertainty includes the jet scale factor (JSF) and b-jet scale factor (bJSF) contributions (when applicable), while the sum of the remaining systematic uncertainties is reported separately. The JSF and bJSF contributions are statistical in nature and apply to analyses performing in-situ (top quark pair based) jet energy calibration procedures.

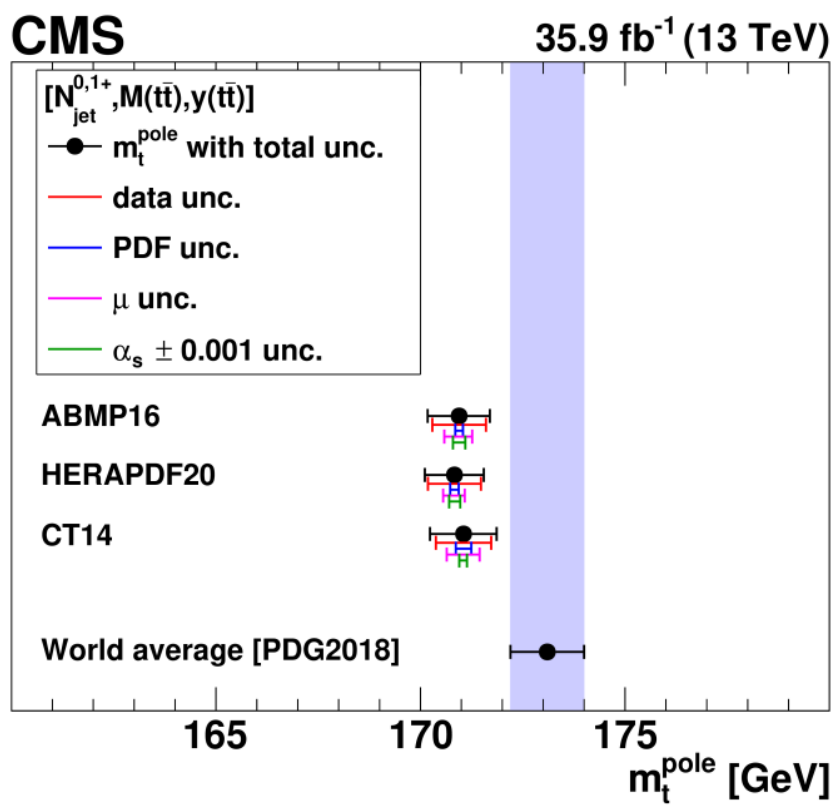

Figure 13: The $m_{t}^{\text {pole }}$ values extracted at NLO using different PDFs. The contributions to the total uncertainty arising from the data, PDF, scale, and $\alpha \mathrm{S}(\mathrm{mZ})$ uncertainties are shown separately. The world average value $m_{t}^{\text {pole }}=173.1 \pm \pm 0.9 \mathrm{GeV}$ from Ref. [18] is shown for reference. 


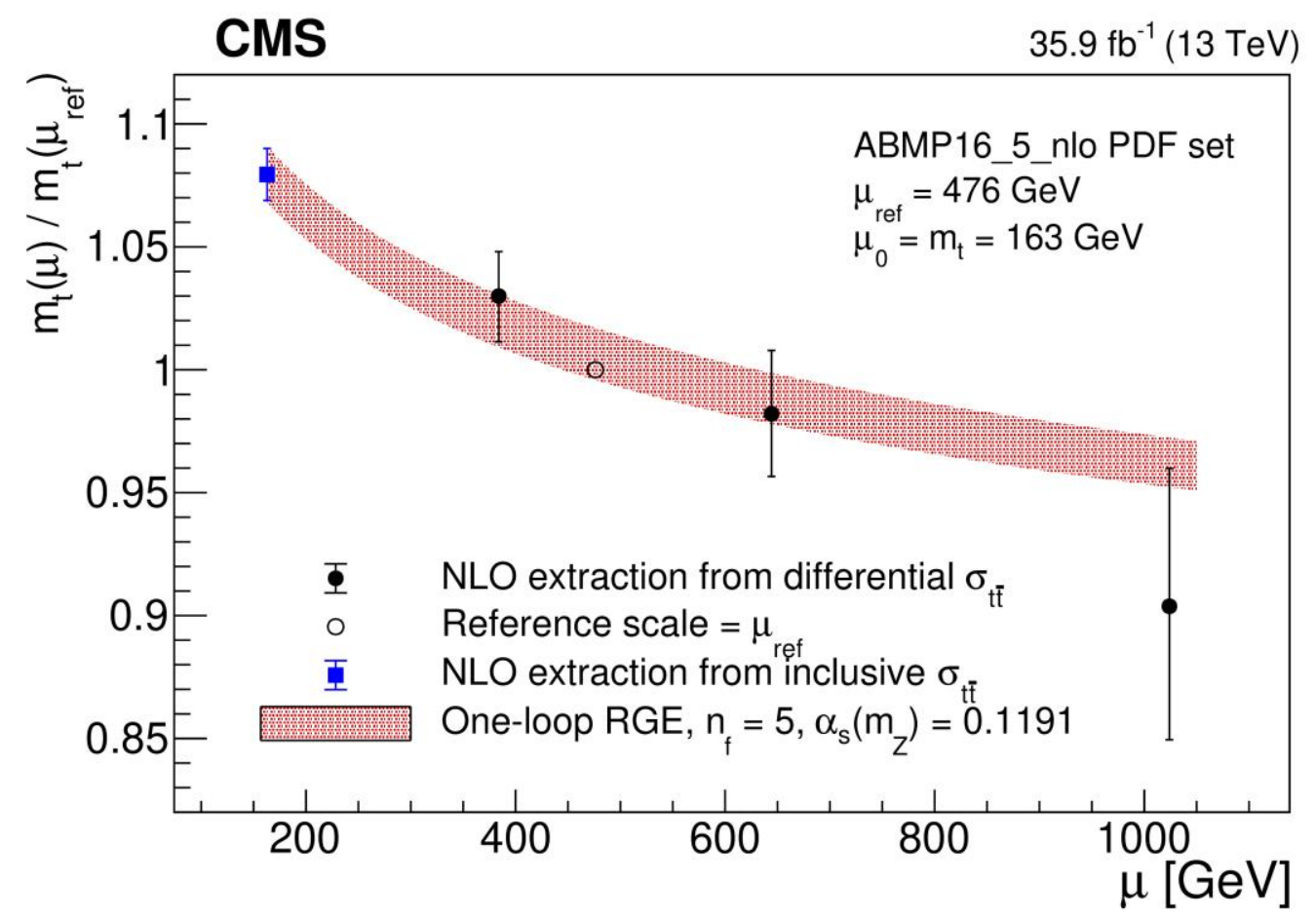

Figure 14: The uncertainty in $m_{t}^{\text {incl. }}\left(m_{t}\right)$ is evolved from the initial scale $\mu_{0}=m_{t}=163$ $\mathrm{GeV}$ using the same RGE prediction.

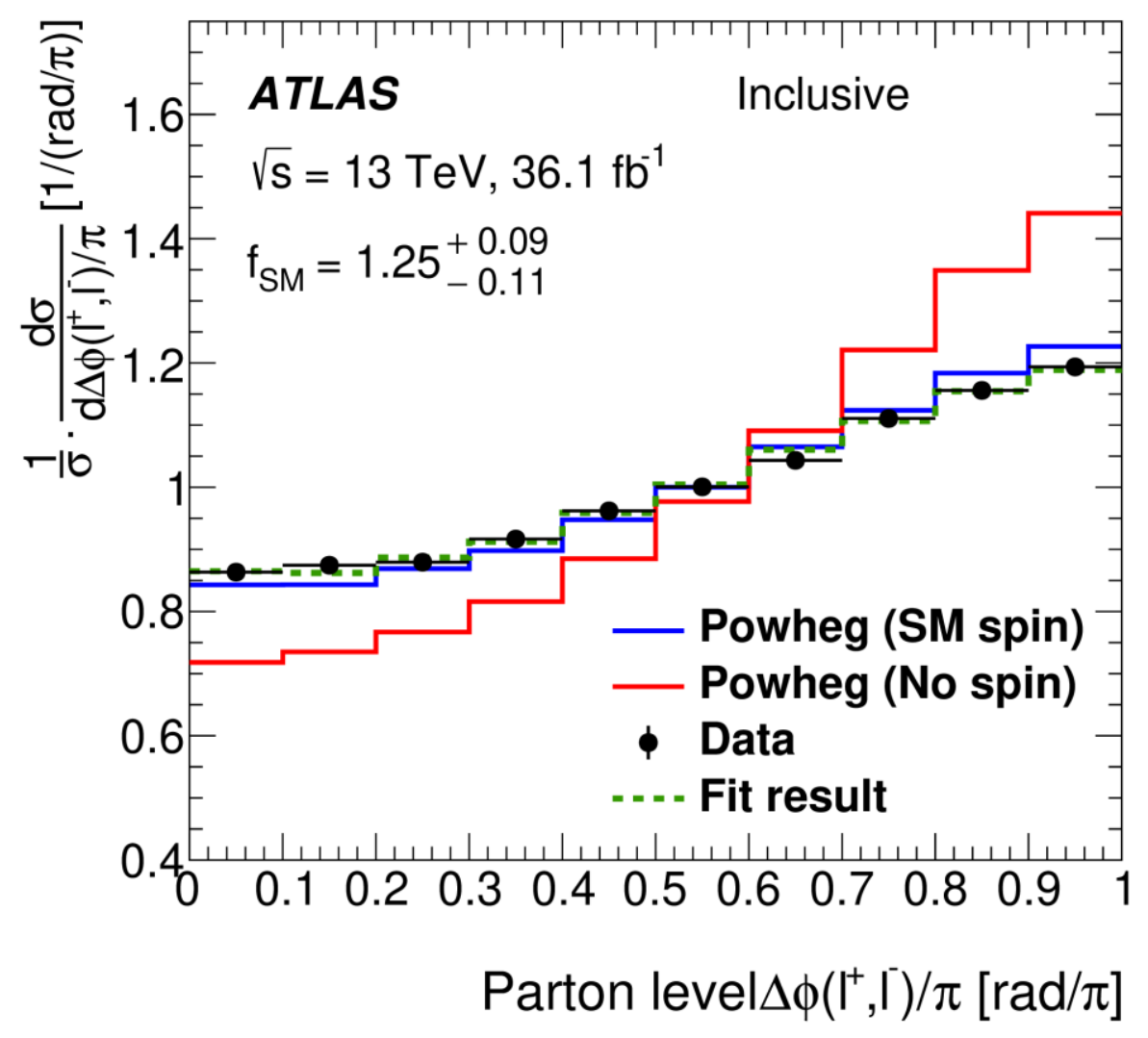

Figure 15: Results of the fit of hypothesis templates to the unfolded data showing the $\Delta \varphi$ distribution for the inclusive selection. 


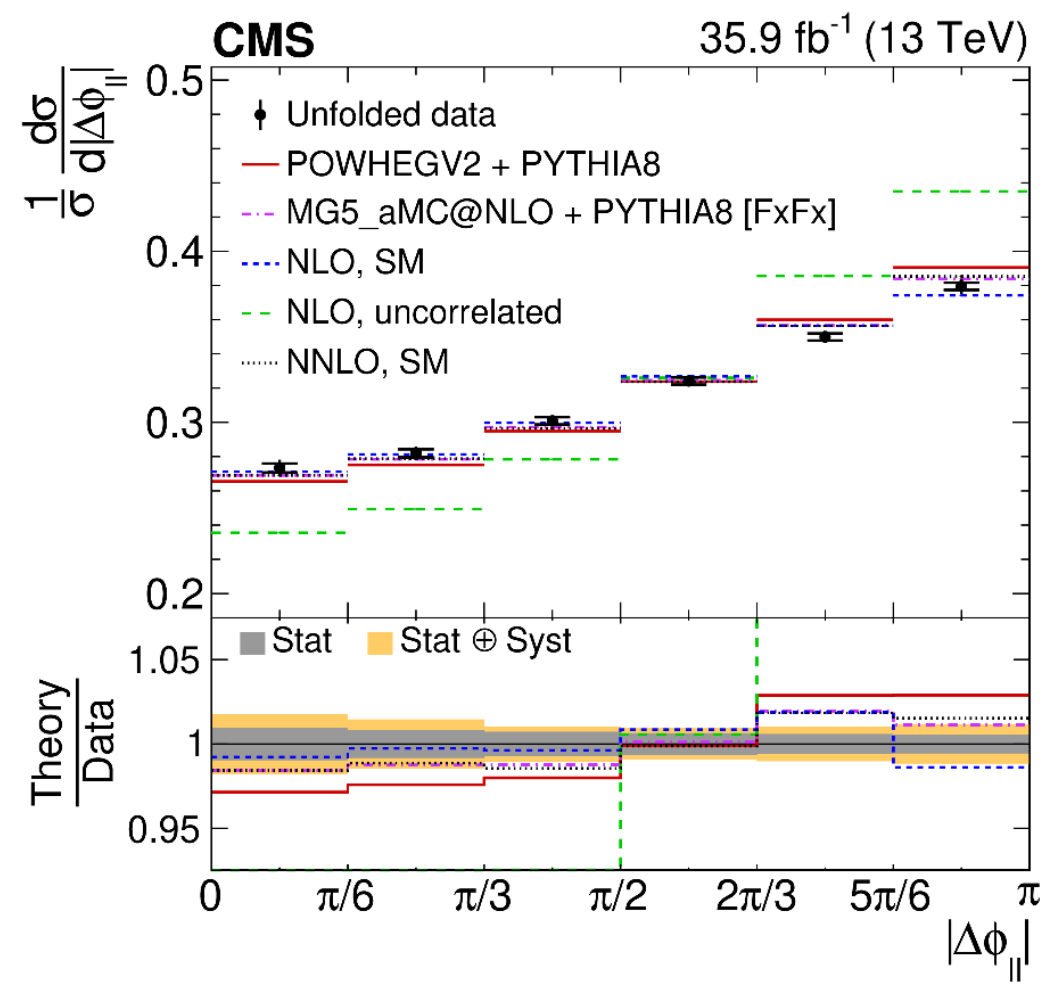

Figure 16: Unfolded data (points) and predicted (horizontal lines) normalized differential cross sections for the diagonal spin correlation observables (first two rows) and the laboratoryframe observables (bottom row). The vertical lines on the points represent the total uncertainties, with the statistical components indicated by horizontal bars. The ratios of various predictions to the data are shown in the lower panels.

\subsection{Top decay width and top pair charge asymmetry}

ATLAS published the direct measurement of the top decay width $\Gamma_{\mathrm{t}}$ in dilepton events with full Run 2 data [22]. In this analysis, data is compared to MC templates with different $\Gamma_{\mathrm{t}}$ assumptions. The measured width is $\Gamma_{\mathrm{t}}=1.9 \pm 0.5 \mathrm{GeV}$.

Top pair charge asymmetry is also measured in ATLAS using full Run2 data [23]. The inclusive $t \bar{t}$ charge asymmetry is measured as $A_{C}=0.0060 \pm 0.0015$ (stat+syst), which is $4 \sigma$ deviation from zero.

\section{Search new physics with top events}

In SM, quark flavours can only change at tree level via charged currents ( $\mathrm{W}+/$ - bosons). Flavour changing neutral currents (FCNC) processes occur via loops in the SM, highly suppressed by GIM mechanism. An observation of FCNC would be unambiguous evidence of BSM. Both ATLAS and CMS have done extensive searches for FCNC processes using top quark decays. A summary of the results can be found in Figure 17.

Local non-conservation of charged lepton flavor is allowed in many BSM scenarios, e.g. minimal extension of the SM explaining neutrino mass. Latest results from ATLAS use $79.8 \mathrm{fb}^{-1}$ data collected from 2015 to 2017 to search for $t \rightarrow l^{ \pm} l^{\prime \mp} q$ decay in $t \bar{t}$ with the other top decays semi-leptonically [24]. Binned maximum-likelihood fit on BDT discriminant is used to test for 
the presence of the signal events. The observed exclusion on cLFV decay branching ratio is $B\left(t \rightarrow l^{ \pm} l^{\mp} q\right)<1.85 \times 10^{-5}$ (observed).

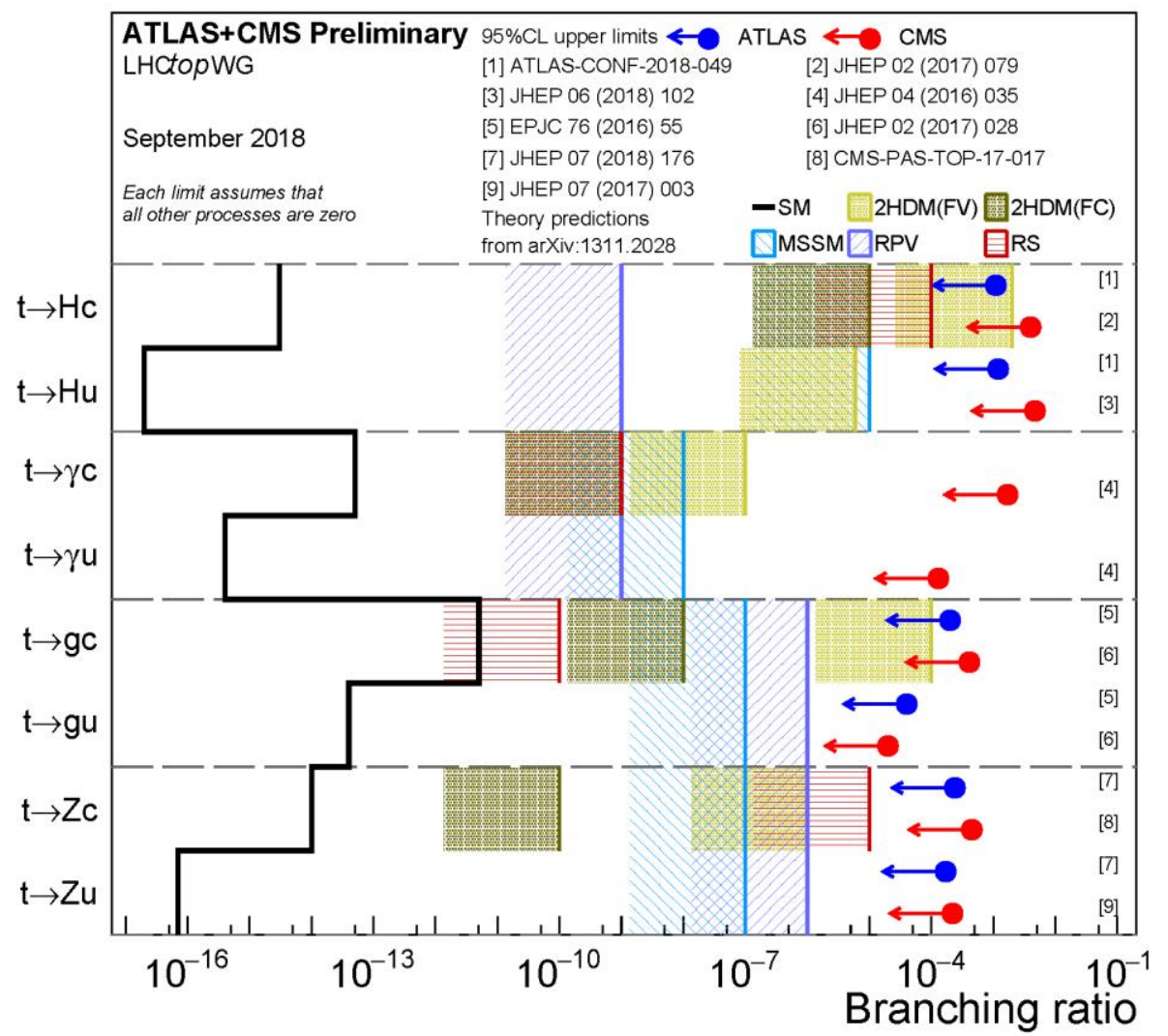

Figure 17: Summary of the current $95 \%$ confidence level observed limits on the branching ratios of the top quark decays via flavour changing neutral currents to a quark and a neutral boson $\mathrm{t}->\mathrm{Xq}(\mathrm{X}=\mathrm{g}, \mathrm{Z}, \gamma$ or $\mathrm{H} ; \mathrm{q}=\mathrm{u}$ or $\mathrm{c})$ by the ATLAS and CMS Collaborations compared to several new physics models.

\section{Summary}

LHC Run 2 data is taking a central stage in top physics studies, a broad range of new results were updated by ATLAS and CMS. This includes precision measurements of top productions and decays, top quark property measurements and improved limits on various new physic searches. Data results are generally consistent with theoretical predictions and no obvious evidence of new physics is observed. In future, more top quark physics will emerge will full Run 2 data and many measurements and searches are expected to be improved with increased statistics and better control of the systematics. 


\section{References}

[1] CMS Collaboration, Measurement of the top quark pair production cross section in dilepton final states containing one $\tau \tau$ lepton in pp collisions at $\sqrt{s}=$ $13 \mathrm{TeV}$, accepted by JHEP, arXiv:1911.13204

[2] ATLAS Collaboration, Measurement of the $t \bar{t}$ production cross-section and lepton differential distributions in e $\mu$ dilepton events from pp collisions at $\sqrt{s}=13 \mathrm{TeV}$ with the ATLAS detector, ATLAS-CONF-2019-041

[3] ATLAS Collaboration, Measurements of $t \bar{t}$ differential cross-sections of highly boosted top quarks decaying to all-hadronic final states in pp collisions at $\sqrt{s}=13 \mathrm{TeV}$ using the ATLAS detector, Phys. Rev. D 98 (2018) 012003

[4] CMS Collaboration, Measurements of $t \bar{t}$ differential cross sections in proton-proton collisions at $\mathrm{s} \sqrt{s}=13 \mathrm{TeV}$ using events containing two leptons, JHEP 02 (2019) 149

[5] CMS Collaboration, Measurement of $t \bar{t}$ normalised multi-differential cross sections in pp collisions at $\sqrt{s}=13 \mathrm{TeV}$, and simultaneous determination of the strong coupling strength, top quark pole mass, and parton distribution functions, Submitted to Eur. Phys. J. C, arXiv:1904.05237

[6] ATLAS and CMS Collaborations, Combinations of single-top-quark production cross-section measurements and $\left|f_{L V} V_{t b}\right|$ determinations at $\sqrt{s}=7$ and $8 \mathrm{TeV}$ with the ATLAS and CMS experiments, JHEP 05 (2019) 088

[7] CMS Collaboration, Observation of single top quark production in association with a $\mathrm{Z}$ boson in proton-proton collisions at $\sqrt{s}=13 \mathrm{TeV}$, Phys. Rev. Lett. 122 (2019) 132003

[8] ATLAS Collaboration, Measurement of the production cross-section of a single top quark in association with a $\mathrm{Z}$ boson in proton-proton collisions at $13 \mathrm{TeV}$ with the ATLAS detector, Phys. Lett. B 780 (2018) 557

[9] CMS Collaboration, Measurement of top quark pair production in association with a $\mathrm{Z}$ boson in proton-proton collisions at $\sqrt{s}=13 \mathrm{TeV}$, accepted by JHEP, arXiv:1907.11270

[10] ATLAS Collaboration, Measurements of inclusive and differential fiducial cross-sections of tt $\gamma$ production in leptonic final states at $\sqrt{s}=13 \mathrm{TeV}$ in ATLAS, Eur. Phys. J. C 79 (2019) 382

[11] ATLAS Collaboration, Search for four-top-quark production in the single-lepton and opposite-sign dilepton final states in pp collisions at $\sqrt{s}=13 \mathrm{TeV}$ with the ATLAS detector, Phys. Rev. D 99 (2019) 052009

[12] CMS Collaboration, Search for production of four top quarks in final states with same-sign or multiple leptons in proton-proton collisions at $\sqrt{s}=13$ TeV, Eur. Phys. J. C 80 (2020) 75

[13] ATLAS Collaboration, Measurements of fiducial and differential cross-sections of tt $^{-}$production with additional heavy-flavour jets in proton-proton collisions at $\sqrt{s}=13 \mathrm{TeV}$ with the ATLAS detector, JHEP 04 (2019) 046

[14] CMS Collaboration, Measurement of the $t \bar{t} b \bar{b}$ production cross section in the all-jet final state in pp collisions at $\sqrt{s}=13 \mathrm{TeV}$, accepted for publication in Phys. Lett. B, arXiv:1909.05306

[15] ATLAS Collaboration, Measurement of the top quark mass in the $\mathrm{tt}^{-} \rightarrow$ lepton+jets channel from $\sqrt{s}=8$ TeV ATLAS data and combination with previous results, Eur. Phys. J. C79 (2019) 290

[16] CMS Collaboration, Measurement of the top quark mass with lepton+jets final states using pp collisions at $\sqrt{s}=13$ TeV, Eur. Phys. J. C 78 (2018) 891 
[17] ATLAS Collaboration, Measurement of the top-quark mass int $\bar{t}+1$-jet events collected with the ATLAS detector in pp collisions at $\sqrt{s}=8 \mathrm{TeV}$, JHEP 11 (2019) 150

[18] Particle Data Group, Review of Particle Physics, Phys. Rev. D 98, 030001

[19] CMS Collaboration, Running of the top quark mass from proton-proton collisions at $\sqrt{s}=13 \mathrm{TeV}$, Accepted for publication in Phys. Lett. B, arXiv:1909.09193

[20] ATLAS Collaboration, Measurements of top-quark pair spin correlations in the e $\mu$ channel at $\sqrt{s}=13$ TeV using pp collisions in the ATLAS detector, submitted to EPJC, arXiv:1903.07570

[21] CMS Collaboration, Measurement of the top quark polarization and $\mathrm{tt}^{-}$spin correlations using dilepton final states in proton-proton collisions at $\sqrt{s}=13$ TeV, Phys. Rev. D 100 (2019) 072002

[22] ATLAS Collaboration, Measurement of the top-quark decay width in top-quark pair events in the dilepton channel at $\sqrt{s}=13 \mathrm{TeV}$ with the ATLAS detector, ATLAS-CONF-2019-038

[23] ATLAS Collaboration, Inclusive and differential measurement of the charge asymmetry in $t \bar{t}$ events at $13 \mathrm{TeV}$ with the ATLAS detector, ATLAS-CONF-2019-026

[24] ATLAS Collaboration, Search for charged lepton-flavour violation in top-quark decays at the LHC with the ATLAS detector, ATLAS-CONF-2018-044 